six operations remain which were performed in the presence of glycosuria. Three operations for epithelioma have been performed, with one death. The fatal case is mentioned by Smith and Durham. The operation was an extensive oneamputation of the whole penis. Sloughing of the wound and gangrene of the left foot brought about a fatal result on the tenth day. The other two operations were slighter, one (Claisse and Durrieux) being a mere circumcision for epithelioma of the prepuce following balano-posthitis. The patient had previously undergone lithotrity for a phosphatic calculus and had been diabetic for eight years. Becker in his paper on Anæsthesia in Diabetes refers to a fatal suprapubic cystotomy for cystitis complicating epithelioma of the bladder and stricture. Sugar was absent the day before the operation, but the day after it amounted to 7 per cent. The patient succumbed on the third day from coma. After a catheterisation under chloroform at St. Bartholomew's Hospital, a man, aged 60 years, died on the third day. The urine contained much sugar. Post mortem a false passage was found in the membranous urethra. In the last two cases the supervention of coma seems to have been due to the anæsthetic. Fort has recorded an interesting case in which, after electrolysis of a stricture in a young man, polyuria appeared the same day, the amoun on the fourth day being eight litres, and in which there was over a kilogramme of sugar. In six months the patient died from diabetes. There had been no sign or symptom of diabetes previously to the operation. This case, taken in conjunction with some others that have been published, raises the question of the possibility of the induction of diabetes through an operation. (Compare the case in which ligature of the femoral artery was performed for aneurysm of the popliteal.) But it must always be remembered that diabetes may be latent. Thus of six operations on five subjects three died, giving an operation mortality of 50 per cent., but no special precautions had been taken in two against coma. (Vide Table V.)

Abdominal Operations other than on the Generative Urgans or Bladder.

Two herniotomies for strangulated hernia have been published; both ended fatally. One patient, a woman, aged 56 years, was known to have been suffering from diabetes for two years. At the time of the operation there was an odour of apples about the breath which pervaded the room and the ferric chloride reaction was present. The herniotomy was done under cocaine; the next day the patient seemed better but coma followed and closed the scene in 36 hours after the operation. The other patient was a man in whom strangulation had existed for four or five days. At the time of the operation the gut was found to be gangrenous; it was therefore left in the wound, to which gangrene spread, the patient dying from sepsis. Barker has recently recorded three abdominal operations on diabetics." In one case four and a half inches of the transverse colon were removed for a malignant growth. The wound did not run a quite aseptic course and later a piece of the mesocolon sloughed, leaving a temporary fæcal fistula. Recovery, however, was complete eventually. General treatment was adopted but no sodium bicarbonate was administered. In another colotomy for complete obstruction was performed. No repair took place and coma ensued. The cause of the obstruction was carcinoma recti. The third patient underwent the operation for the radical cure of an umbilical hernia. Sugar, which had not been observed previously, appeared the next day. Repair was bad but healing eventually took place. A gastro-jejunostomy for cancer of the stomach proved fatal in two days, though not from diabetic complications; 0.3 per cent. of sugar was present. Fisk has recorded an appendicectomy in a man, aged 50 years, on the second day of an acute attack. The wound healed slowly by granulation. The patient had been diabetic for years (compare Table I., cases of temporary glycosuria accompanying appendicitis). Three pancreatic cysts in diabetics have been drained; in each case the patient survived the immediate effects of the operation but died some months after. In Churton's case the cyst was drained in December, 1892 ; the wound became a mere sinus by July, 1893, but under improper and poor diet out of hospital the health of the patient became worse and he was readmitted in September with a freely discharging wound. $\mathrm{He}$ went gradually down hill and died in January, 1894, 13 months after the original operation. Post mortem there was free retroperitoneal suppuration and the ureter was found to be ruptured. In Pagenstecher's case (mentioned by Körte) a man, aged 40 years, had a pancreatic cyst of the size of a child's head. The urine contained 4.5 per cent. of sugar. This was drained on Dec. 12th, 1888 . On his discharge from hospital the wound was a mere sinus. Death from diabetes occurred one and a half years after the operation. In Bull's case an emaciated man, aged 45 years, in whose urine 4.5 per cent. of sugar was present, underwent laparotomy on July 10th, 1886, at which the cyst was fixed. It was opened and drained on the 26th, 118 ounces of fluid being evacuated. By Sept. 12th the patient had gained $12 \frac{1}{2}$ pounds in weight. Up to this date there had been no special dietetic or medical treatment, the percentage of sugar being 5 . On the 18th he was dieted and was put on codeine. The percentage of sugar fell to 1.25. On Nov. 13th he left the hospital with a sinus two and a half inches deep. Two weeks later be died suddenly. There was no necropsy. He had discontinued diet and medicine on leaving the hospital. The number of pancreatic cysts recorded with sugar in the urine is quite small. These three cases show that even pancreatic diabetes is no bar to operation, though the final prognosis is less good if general precautions be neglected. Noble of Philadelphia has published an account of a radical cure of a ventral hernia. in a woman, aged 60 years, the hernia being the sequela of the removal of the appendages some years previously. The amount of sugar in her urine diminished under the influence of codeine and strychnine. Recovery was complete. 18 months later her urine was free from sugar. Three years later sbe was still well though continuing general treatment. In a radical cure of an umbilical hernia recorded by Reynier slight disunion of the wound occurred but recovery followed. Two other radical cures by the same observer were successful. Dr. Fisk has kindly furnished the notes of a radical cure by Bassini's method for a pro-peritoneal hernia in a youth, aged 17 years. Recovery was perfect. The percentage of sugar varied from 1.5 to $2 \cdot 5$. This is the youngest diabetic patient upon whom an operation seems to have been performed. Thus, of 15 operations on 15 patients 11 recovered, which gives a percentage of 73.3 . As, however, three cases were moribund or nearly so at the time of the operation the percentage of recoveries is unduly low. It must be remembered that several laparotomies are to be found in the section on operations on the female generative organs. There are also some cases in Class 1 in which, though apparently diabetic, yet after operation sugar permanently vanished. (Fide Table VI.)

$$
\text { (To be concluded.) }
$$

\section{CASE OF POISONING BY MORPHIA INJECTION TREATED BY INFU- SION OF SALT SOLUTION.}

\section{By EDWARD F. WILLOUGHBY, M.D. LoND.}

AT about 1 P.M. on Sept. 15th, 1901, I was called to see a woman who had, as I learnt, taken eight grains of sulphate of morphia in three hypodermic injections less than half an hour before. She had not acquired any tolerance, having had it administered on one occasion only, when it caused such violent sickness that she refused to have it repeated, preferring to endure the pain from which she was then suffering after an sbdominal operation. I was soon joined by the locum. tenent of her ordinary medical attendant who was out of town. but he, being a young man, left the conduct of the case to me. On our arrival the patient was still sericonscious, but within 10 minutes she sank into a state of profound narcosis from which it was impossible to rouse her. During the first hour we administered three injections of onefiftieth of a grain of sulphate of atropine and one-tenth of a grain of nitrate of strychnine without any perceptible effect, except that the strychnine excited spasms from every 10 to 20 minutes. The rigidity of the muscles, which lasted for from three to five minutes each time, greatly interfered with the artificial respiration which soon-became necessary. We gave also at least a dozen injections of 20 minims of ether, with what benefit we could not say. The partner of the patient's own medical attendant joined us later, bringing a galvanic battery. Whenever we desisted 
from our efforts at artificial respiration the natural respiration ceased and it was four hours before we were able to discontinue it. Though by this time natural respiration was re-established and the spasms had passed off the patient's pupils were contracted to pin-points, her complexion was dusky, and the narcosis was so profound that the application of the strongest possible interrupted current to the soles of both feet did not excite the slightest movement even of the toes. She had not passed any urine and I drew off only about an ounce with the catheter. I then suggested the subcutaneous injection of the physiological salt solution as recommended first by Sahli of Berne. The partner was somewhat doubtful and averse to the procedure and was absent for an hour in quest of the apparatus. But when about a litre had been infused by gravitation into the subcutaneous tissue of the flank the effect was almost immediate and was striking. Within 20 minutes the duskiness and coldness of the skin had given place to a natural colour and warmth and the patient had so far recovered sensibility and consciousness as to struggle violently against the mechanical and electrical stimulation employed to keep her awake. She could answer questions intelligibly and she recognised the other medical men but she knew me to be a stranger and continually begged to be left alone.

I felt certain that the startling improvement following so soon after the subcutaneous infusion of 30 ounces of salt solution was owing to the impulse given by the increased intra-vascular tension to the gastric and intestinal exudation and consequently to the elimination of the morphia, which ordinarily is excreted mainly in the bowel and little, if at all, by the kidneys; but that a certain proportion of the drug being probably re-absorbed the complete removal of so large a quantity would be long and tedious. I knew the action of potassium permanganate in the breaking up of morphia, even, as Dr. A. P. Luff had shown in the presence of oxidisable matter as vomit, and I should have had instant recourse to this antidote had the poison been swallowed. But it occurred to me that since the drug was now passing out into the alimentary canal it might be destroyed as fast as it appeared by contact with the permanganate and reabsorption might be prevented. I therefore mixed about a quart of a dilute solution, instructing the nurse to give the patient a wineglassful, alternately with her nourishment, every quarter of an hour or 20 minutes through the night until the contraction of the pupils had passed off. I did not see the patient again but was informed by her medical attendant that by 9 A.M. on the next day her condition was quite normal but for the sense of fatigue and soreness consequent on a score of hypodermic punctures and four hours of sustained artificial respiration.

In looking back on such a case one naturally asks oneself to which, if any, of the means employed the success was really due, which were superfluous, and whether any were useless or possibly even hurtful. We used hypodermic injections of atropine, strychnine, and ether, secundum artem, in deference to usage and because nothing could be taken per os, but I cannot honestly say that they were of any benefit except the ether which could not but act as a general stimulant. The respiration ceased again and again while the action of the atropine was visibly antagonising that of morphia on the pupils and the tonic contractions of the muscles caused by the strychnine made the work of artificial respiration extremely laborious. This alone kept the patient in life but that was all, for when at the end of four or five hours from the taking of the poison natural respiration seemed to be re-established she was still absolutely insensitive to pain or to electric shocks and deglutition was still impossible. The effect, however, of the saline subcutaneous infusion was such as to admit of no doubt and it was startling in the rapidity of its manifestation. So long as no fluids could be swallowed no urine was secreted and there was no reason why the morphia should be eliminated by the bowel until the intra-vascular pressure was raised by the infusion. Then, even, a long and anxious time, not wholly free from danger, would have ensued during which much of the drug would have been re-absorbed and circulated again through the brain but for its destruction by the permanganate pari passu with its first elimination. In short, I look on the injections as useless, on the artificial respiration as having served to keep the patient alive until means could be taken for washing out the poison, the saline infusion as having effected this, and the permanganate as having greatly aided the result by preventing re-absorption.

Green Lanes, N.

\section{AN INTERESTING PHENOMENON OCCUR- RING IN TACHYCARDIA.}

\author{
BY HERBERT D. EVERINGTON, M.B., L.R.C.P. LOND.,
} M.R.C.S. ENG.

LATE RESIDENT MEDICAL OFFICER, ROYAL HOSPITAL FOR CHIMDREN AND WOMEN, WATERLOO-ROAD, S.E.

A MARRIED woman, aged 36 years, was admitted into the Royal Hospital for Children and Women Waterloo-road on August 3rd, 1900, suffering from articular pains and swellings which she had had for some days previously. On admission she looked decidedly ill. The face was puffy and congested, the tongue was dry and furred down the centre, and the pulse was of full volume, high tension, and "bounding," the beats numbering 128 to the minute, no irregularity being noted. The temperature was $103^{\circ} \mathrm{F}$. Nothing abnormal was found in the chest or the abdomen. The left knee and wrist were acutely tender and were much swollen. The patient was placed on a diet of milk and was. given 10 grains each of the bicarbonate and salicylate of soda with half a drachm of sal volatile every four hours. Her condition remained much the same for some days and on the 7th the alkaline salicylate mixture was replaced by doses of 10 grains of salicylic acid given in cachets every six hours. The diet was made more generous. Up to the 9 th no undue acceleration of the pulse was noted, the range varying more or less with the temperature from 92 to 112 per minute. On the night of the 9th, however, this moderate pulse-rate had given place to one of extreme rapidity. The frequency could no longer be counted at the wrist, the pulse being too rapid and "running." By means of the stethoscope, however, over the heart the beats could be distinguished and were found to number 224 to the minute. No complaint was made with reference to the heart though the patient complained of the pain in the limbs. The salicylic acid was immediately discontinued and five minims of tincture of digitalis with 30 minims of sal volatile were given and repeated every four hours. During the early hours of the morning the pulse maintained an extremely rapid rate and was numbering 210 when the morning round was made. A prescription was ordered containing 10 minims of tincture of digitalis, five minims of tincture of nux vomica, half a drachm of aromatic spirit of ammonia, 10 minims of spirit of chloroform, and distilled water to make up one ounce. This was given every four hours. On the afternoon of the 10th the patient was examined by Dr. Guthrie Rankin who found no evidence of organic disease of the heart and no dilatation whatever. The heart beats were counted at about 220 per minute. During his examination Dr. Rankin ordered the patient to take deep breaths and after several of these inspirations it was found that the heart beats had dropped to 124 in the minute, or roughly a fall of 100 beats. A mixture of 15 grains each of the bromide and salicylate of sodium was given every four hours, alternating with the digitalis mixture above mentioned. The pulse was not noticed to be unduly accelerated for several days but the temperature remained high, averaging $100^{\circ}$. The bromide was discontinued on the 18th. The patient gradually went down hill and the tongue became extremely dry and foul, sordes developing on the teeth and lips. On the 22nd she presented a decidedly "typhoid" appearance. Soon after midnight on the 25th the pulse had again risen and it numbered 192 per minute. On taking some deep breaths the rapidity fell immediately to 108 . On the night of Sept. 2nd tachycardia was again present-202 beats per minute-but this time the rapidity could not be reduced by deep inspirations as heretofore, nor did they have such an effect subsequently. The patient lost ground continually. On the 5 th the highest rate of pulse was noted, the beats numbering no less than 234 per minute at 2.25 P. M., when the temperature was $103^{\circ}$. From being in an extremely critical condition the patient began to show signs of improvement and by the 24th she was out of immediate danger. No further tachycardia was recorded. The knee and wrist improved but never returned to their natural state. The patient's general health became very much better and she finally left the hospital in December.

There are one or two extremely interesting points in connexion with this case. Leaving on one side the question of diagnosis of the articular trouble which presented much difficulty and turning to the condition of tachycardia the 